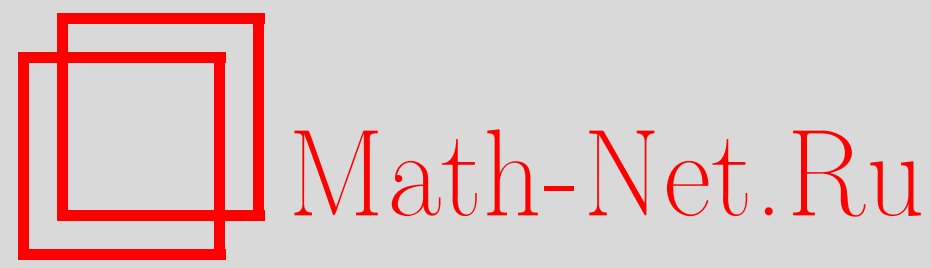

Д. Анер, В. В. Дякин, В. Я. Раевский, С. Риттер, О спектральных свойствах операторов теории гармонического потенциала, Матем. заметки, 1996, том 59, выпуск $1,3-11$

DOI: https://doi.org/10.4213/mzm1689

Использование Общероссийского математического портала MathNet.Ru подразумевает, что вы прочитали и согласны с пользовательским соглашением

http://www . mathnet.ru/rus/agreement

Параметры загрузки:

IP : 54.164 .48 .24

26 апреля 2023 г., $11: 08: 29$

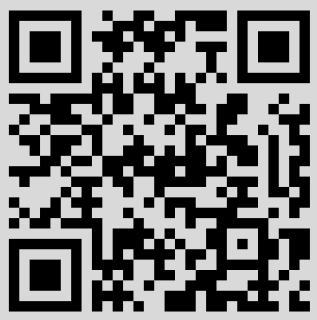




\title{
О СПЕКТРАЛЬНЫХ СВОЙСТВАХ ОПЕРАТОРОВ ТЕОРИИ ГАРМОНИЧЕСКОГО ПОТЕНЦИАЛА
}

\author{
Дж. Анер, В.В. Дякин, В.Я. Раевский, Ст. Риттер
}

\section{§ 1. Введение}

Пусть $\Omega$ - односвязная конечная область в $\mathbb{R}^{3}$, ограниченная ляпуновской поверхностью $S$. Предметом данной работы является исследование спектральных свойств операторов классической теории потенциала

$$
\begin{aligned}
(T \sigma)(x) & :=\int_{S} \sigma(y)|x-y|^{-1} d S_{y} \\
(B \varphi)(x) & :=\int_{S} \varphi(y) \frac{\partial|x-y|^{-1}}{\partial n_{x}} d S_{y}, \\
\left(B^{*} \psi\right)(x) & :=\int_{S} \psi(y) \frac{\partial|x-y|^{-1}}{\partial n_{y}} d S_{y}
\end{aligned}
$$

действующих в пространстве $L_{2}(S)$. Оператор $T$ представляет собой значение на $S$ потенциала простого слоя, $B$ - оператор прямого значения на $S$ нормальной производной потенциала простого слоя, а $B^{*}$ - сопряженньй ему оператор прямого значения на $S$ потенциала двойного слоя. Известно (см. $[1$, с. 160]), что ненулевые точки спектра компактных операторов $B$ и $B^{*}$ суть вещественные простые полюсы их резольвент, содержащиеся в полуинтервале $[-2 \pi, 2 \pi)$, а соответствуюшие собственные функции непрерьвны. Однократному собственному значению $\mu_{0}=-2 \pi$ операторов $B$ и $B^{*}$ отвечают такие собственные функции $\sigma_{0}$ и $c_{0}$, что $\int_{S} \sigma_{0} d S \neq 0$, a $c_{0}=$ const (см. [2, с. 276], [3, с. 334]). Кроме того, оператор $B$ является квазиэрмитовым (см. [4, с. 394]), т.е. существует такой положительньй самосопряженньй оператор $T$ (таковьм является компактньй оператор $T$ в (1)), что $T B=B^{*} T$ (см. [5]). Пусть $\left\{\mu_{n}\right\}$ - последовательность ненулевых собственных значений $B$ (а потому и $\left.B^{*}\right)$, а $E(\mu, B)$ означает конечномерное собственное подпространство оператора $B$, отвечающее собственному значению $\mu \in\left\{\mu_{n}\right\}$. Тогда следствием квазиэрмитовости является

Работавыполнена при финансовой поддержке Российского фонда фундаментальных исследований. 
полнота системы собственных функций оператора $B^{*}$, ортогональность по энергии оператора $T$ (т.е. в скалярном произведении $(T \cdot, \cdot))$ собственных функций $B$, отвечающих различным собственньм значениям, а также соотношения (см. [5]): $E\left(\mu, B^{*}\right)=T(E(\mu, B)), \varphi \in \operatorname{Ker} B \Leftrightarrow T \varphi \in \operatorname{Ker} B^{*}$. Если $S \in C^{\infty}$, то все операторы в (1) являются псевдодифференциальными (ПДО) порядка $(-1)$, т.е. ограниченным образом действуют из соболевского пространства $H^{r}(S)$ в $H^{r+1}(S)$ для всех $r \in R$, а оператор $T$ - эллиптический ПДО, имеющий обратный, которьй может быть расширен до непрерьвного из $L_{2}(S)$ на $H^{-1}(S)$ (если $S \in C^{\infty}$, то $T^{-1}$ отображает $H^{r}(S)$ на $H^{r-1}(S)$ для всех $r \in \mathbb{R}($ см. [6], [7, с. 151])). Таким образом, спектр компактного самосопряженного положительного оператора $T$ состоит из положительных конечнократных собственных значений и нуля, принадлежащего его непрерьвной части. Изложенные свойства операторов $B$ и $B^{*}$ позволяют заключить, что их спектр состоит из системы конечнократных собственных значений из полуинтервала $[-2 \pi, 2 \pi)$, которым соответствуют бесконечногладкие собственные функции, и нуля.

В теории потенциала долгое время остается открытым вопрос о классификации нуля в спектре операторов $B$ и $B^{*}$ (см. [8]): какой части спектра (точечной $\sigma_{p}$, непрерывной $\sigma_{c}$ или остаточной $\sigma_{r}$ ) этих операторов может принадлежать нуль? В данной работе получен частичный ответ на этот вопрос. Кроме того, дано обобщение классического результата Племеля 1911 года о принадлежности спектров операторов $B$ и $B^{*}$ полуинтервалу $[-2 \pi, 2 \pi)$, а именно, доказано, что любая точка полуинтервала является собственным значением этих операторов при подходящем выборе поверхности. Для полноты изложения в $§ 3$ мы включили краткое изложение некоторых результатов, полученньх нами в [9]. В заключительной части доказан ряд новых спектральных свойств операторов $B$ и $B^{*}$ для эллипсоидальных поверхностей.

\section{§2. Предварительные результаты}

Свойство квазиэрмитовости оператора $B$ позволяет сформулировать следующее утверждение.

Teopema $1.0 \notin \sigma_{r}\left(B^{*}\right)$.

ДоКАЗАТЕЛЬСТВо. Предположим противное, т.е. что $0 \in \sigma_{r}\left(B^{*}\right) \Rightarrow$ $\operatorname{dim} \operatorname{Ker} B \neq 0$. Пусть $\varphi \in \operatorname{Ker} B$,

$$
\begin{aligned}
\varphi \neq 0 & \Rightarrow T B \varphi=0 \Rightarrow B^{*} T \varphi=0 \Rightarrow T \varphi \in \operatorname{Ker} B^{*} \\
& \Rightarrow 0 \in \sigma_{p}\left(B^{*}\right) \Rightarrow 0 \notin \sigma_{r}\left(B^{*}\right),
\end{aligned}
$$

что и доказывает утверждение теоремы.

Учитывая этот результат и связь спектров сопряженных операторов (см. [7, с. 34]), легко установить, что “а priori" могут реализовьваться 
только три возможности:

$$
\begin{array}{ll}
0 \in \sigma_{c}(B), & 0 \in \sigma_{c}\left(B^{*}\right), \\
0 \in \sigma_{p}(B), & 0 \in \sigma_{p}\left(B^{*}\right), \\
0 \in \sigma_{r}(B), & 0 \in \sigma_{p}\left(B^{*}\right) .
\end{array}
$$

Известно (см. [8], [10]), что в случае, когда $S$ есть сфера или вытянутый сфероид, то реализуется возможность (2). В работе [8] поднят вопрос: всегда ли нуль содержится в непрерывном спектре операторов $B$ и $B^{*}$ для достаточно гладких поверхностей $S$ ? В следующем параграфе мы дадим отрицательный ответ на это вопрос, приведя пример, когда реализуется возможность (3). Что касается (4), то вопрос остается открытым. Мы получили в этом направлении следующий результат.

TEOpema 2. $0 \in \sigma_{r}(B) \Leftrightarrow \varnothing \neq \operatorname{Ker} B^{*} \backslash\{0\} \subset L_{2}(S) \backslash H^{1}(S)$.

ДоказАтЕЛьСтво. 1. Пусть $0 \in \sigma_{r}(B) \Rightarrow 0 \in \sigma_{p}\left(B^{*}\right) \Rightarrow \operatorname{Ker} B^{*} \neq\{0\}$. Предположим, что $\exists \psi: \psi \neq 0, \psi \in H^{1}(S), B^{*}(\psi)=0$. Поскольку оператор $T$ есть биекция $L_{2}(S)$ на $H^{1}(S)$ (см. [11, с. 87], [12]), то $\exists \varphi \in L_{2}(S)$, $\varphi \neq 0$ :

$$
T \varphi=\psi \Rightarrow B^{*} T \varphi=0 \Rightarrow T B \varphi=0 \Rightarrow B \varphi=0 \Rightarrow 0 \in \sigma_{p}(B)
$$

- противоречие.

2. Пусть $\varnothing \neq \operatorname{Ker} B^{*} \backslash\{0\} \subset L_{2}(S) \backslash H^{1}(S) \Rightarrow 0 \in \sigma_{p}\left(B^{*}\right) \Rightarrow 0 \in \sigma_{r}(B)$, или $0 \in \sigma_{p}(B)$. Исключим вторую возможность. Имеем

$$
\begin{aligned}
0 \in \sigma_{p}(B) & \Rightarrow \exists \varphi \neq 0: B \varphi=0 \Rightarrow \psi:=T \varphi \in H^{1}(S), B^{*} \psi=T B \varphi=0 \\
& \Rightarrow \operatorname{Ker} B^{*} \backslash\{0\} \not \subset L_{2}(S) \backslash H^{1}(S)
\end{aligned}
$$

- противоречие, завершающее доказательство.

Может ли $\operatorname{Ker} B^{*} \backslash\{0\}$ состоять только из “негладких" функций? Отметим, что попытка доказать невозможность (4) должна опираться на специфические свойства операторов $B$ и $B^{*}$, ибо для общих квазиэрмитовых операторов возможность (4) может быть реализована (см. [13]).

\section{§3. Спектральные свойства операторов $B$ и $B^{*}$ для сплюснутого сфероида}

В этом параграффе мы докажем возможность реализации (3), когда $S$ является сплюснутым сфероидом (эллипсоидом вращения). Декартовы координаты $(x, y, z)$ связаны с координатами $(\xi, \eta, \varphi)$ сплюснутого сфероида соотношением (см. $[14$, с. 24]): $x=p \cos \varphi, y=p \sin \varphi, z=d \xi \eta / 2$, где $p^{2}=d^{2}\left(1+\xi^{2}\right)\left(1-\eta^{2}\right) / 4, d$-межфокальное расстояние, $0 \leqslant \xi<\infty$, $-1 \leqslant \eta \leqslant 1,0 \leqslant \varphi<2 \pi$. Координатные поверхности $\xi=$ const есть сплюснутые эллипсоиды врашения вокруг оси $z$. Если в качестве $S$ взять 
такую поверхность, то собственные значения операторов $B^{*}$ и $B$ имеют вид (см. [15]):

$$
\begin{aligned}
\lambda_{n}^{m}(\xi)=-2 \pi\{1 & +2(-1)^{m} \frac{(n-m) !}{(n+m) !} Q_{n}^{m}(i \xi)\left[(n+1) i \xi P_{n}^{m}(i \xi)\right. \\
& \left.\left.-(n-m+1) P_{n+1}^{m}(i \xi)\right]\right\}
\end{aligned}
$$

а соответствующие собственные функции этих операторов

$$
\begin{aligned}
& \left\{\begin{array}{l}
C_{n}^{m}(\eta, \varphi):=P_{n}^{m}(\eta) \cos m \varphi \\
S_{n}^{m}(\eta, \varphi):=P_{n}^{m}(\eta) \sin m \varphi
\end{array}\right. \\
& \left\{\begin{array}{l}
C_{n}^{m}(\xi, \eta, \varphi):=C_{n}^{m}(\eta, \varphi)\left(\xi^{2}+\eta^{2}\right)^{-0.5} \\
S_{n}^{m}(\xi, \eta, \varphi):=S_{n}^{m}(\eta, \varphi)\left(\xi^{2}+\eta^{2}\right)^{-0.5}
\end{array}\right.
\end{aligned}
$$

где $n=0,1,2, \ldots, 0 \leqslant m \leqslant n, P_{n}^{m}$ и $Q_{n}^{m}$ обозначают присоединенные функции Лежандра. Найдем асимптотическое поведение собственных значений (5) при $\xi \rightarrow \infty$ и $\xi \rightarrow 0$.

ЛЕмма 1. $\lambda_{n}^{m}(\xi) \rightarrow-2 \pi /(2 n+1) n p u \xi \rightarrow \infty$.

ДокАЗАТЕльство. Используя асимптотические формулы (см. [16, c. 165])

$$
\begin{aligned}
P_{n}^{m}(i \xi) & =\frac{(2 i \xi)^{n} \Gamma(n+1 / 2)}{\sqrt{\pi} \Gamma(n-m+1)}\left(1+O\left(\xi^{-2}\right)\right) \\
Q_{n}^{m}(i \xi) & =(-1)^{m} \frac{\sqrt{\pi} \Gamma(n+m+1)}{(2 i \xi)^{n+1} \Gamma(n+3 / 2)}\left(1+O\left(\xi^{-2}\right)\right)
\end{aligned}
$$

где Г обозначает гамма-функцию, из (5) после некоторых преобразований получаем

$$
\lambda_{n}^{m}(\xi)=-\frac{2 \pi}{2 n+1}+O\left(\xi^{-2}\right) .
$$

В завершение доказательства отметим, что, как и следовало ожидать, при $\xi \rightarrow \infty$ получаются собственные функции операторов $B$ и $B^{*}$ для сферы.

ЛЕмма 2. $\lambda_{n}^{m}(\xi) \rightarrow 2 \pi(-1)^{n+m+1} n p u \xi \rightarrow 0$.

ДокАЗАТЕльство. Подставляя $z=i \xi$ в формулу для $Q_{n}^{m}(z)$ через гипергеометрическую функцию аргумента $z^{2}$ (см. $[17$, с. 155]), после некоторых преобразований получаем

$$
\begin{aligned}
Q_{n}^{m}(i \xi)= & \exp \left(\frac{i \pi(3 m-n)}{2}\right) \sqrt{\pi} 2^{m-1}\left(-\xi^{2}-1\right)^{m / 2} \\
& \times\left\{-\frac{\Gamma((n+m+1) / 2)}{\Gamma((n-m+2) / 2)}+O(\xi)\right\} .
\end{aligned}
$$


Отталкиваясь от аналогичной формулы для $P_{n}^{m}(z)$ (см. [17, с. 154]), получим

$$
\begin{aligned}
P_{n}^{m}(i \xi)= & 2^{m}\left(-\xi^{2}-1\right)^{-m / 2} \pi^{-1 / 2} \\
& \times\left\{\frac{\cos \left(\pi \frac{n+m}{2}\right) \Gamma\left(\frac{n+m+1}{2}\right)}{\Gamma\left(\frac{n-m+2}{2}\right)}+O(\xi)\right\} .
\end{aligned}
$$

Подставляя (6) и (7) в (5), имеем формулу

$$
\begin{aligned}
\lambda_{n}^{m}(\xi) & \\
= & -2 \pi\left\{1+2^{m} \alpha_{n}^{m} \exp \left(-i \pi \frac{n+m}{2}\right) i(n-m+1) \Gamma\left(\frac{n+m+1}{2}\right) \Gamma\left(\frac{n+m+2}{2}\right)\right. \\
& \left.\times \cos \left(\pi \frac{n+m+1}{2}\right) /\left[\Gamma\left(\frac{n-m+2}{2}\right) \Gamma\left(\frac{n-m+3}{2}\right)\right]\right\}+O(\xi),
\end{aligned}
$$

где для удобства введено $\alpha_{n}^{m}:=(n-m) ! /(n+m) !$.

Теперь нетрудно убедиться, что в случае четности суммы $n+m$ : $\lambda_{n}^{m}(\xi)=-2 \pi+O(\xi)$, а в случае ее нечетности: $\lambda_{n}^{m}(\xi)=2 \pi+O(\xi)$, что и завершает доказательство леммы.

Следующая теорема утверждает, что возможность (3) может реализовываться.

ТЕОрема 3. Существует поверхность $S$ в форме сплющенного сфероида, для которой $0 \in \sigma_{p}(B), 0 \in \sigma_{p}\left(B^{*}\right)$.

ДокАЗАТЕЛЬСТВо. Пусть $n$ и $m$ таковы, что сумма $n+m$ нечетна. Тогда из лемм 1,2 и непрерьвности $\lambda_{n}^{m}(\xi)$ следует, что существует $\xi_{n}^{m} \in(0, \infty)$ такое, что $\lambda_{n}^{m}\left(\xi_{n}^{m}\right)=0$. Утверждению теоремы соответствуют все сплюснутые сфероиды с $\xi=\xi_{n}^{m}$.

Содержание теоремы отвечает на вопрос, поднятый Анером в [8]. В общем случае нельзя утверждать, что для всех гладких поверхностей операторы $B$ и $B^{*}$ инъективны, спектральная характеристика нуля зависит от формы поверхности $S$.

Следующая теорема представляет собой обобщение классического результата Племеля [8], утверждающее о невозможности сужения полуинтервала $[-2 \pi, 2 \pi)$ для собственных чисел $B$ и $B^{*}$.

Теорема 4. Для всех $b \in[-2 \pi, 2 \pi)$ существует гладкая поверхность $S$ такая, что $b$ является собственным значением $B u B^{*}$, определенных на $S$.

ДокАЗАТЕльство. Для $b=-2 \pi$ утверждение справедливо для любой гладкой поверхности, а из лемм 1 и 2 следует, что объединение областей значения функций $\lambda_{1}^{0}(\xi)$ и $\lambda_{2}^{0}(\xi)$ совпадает с интервалом $(-2 \pi, 2 \pi)$, что и завершает доказательство теоремы. 


\section{§4. Некоторые спектральные свойства операторов $B$ и $B^{*}$ для эллипсоидальных поверхностей}

Формула (5), а также аналогичная формула для собственных значений $B$ и $B^{*}$ в случае вытянутого сфероида (см. [10]) могут быть записаны в виде

$$
\lambda_{n}^{m}(\xi)=2 \pi i(-1)^{m}\left(1+\xi^{2}\right) \alpha_{n}^{m} \frac{\partial\left\{P_{n}^{m}(i \xi) Q_{n}^{m}(i \xi)\right\}}{\partial \xi}, \quad \xi \in(0, \infty),
$$

для сплюснутого и

$$
\lambda_{n}^{m}(\xi)=2 \pi\left(\xi^{2}-1\right) \alpha_{n}^{m}(-1)^{m} \frac{\partial\left\{P_{n}^{m}(\xi) Q_{n}^{m}(\xi)\right\}}{\partial \xi}, \quad \xi \in(1, \infty),
$$

для вытянутого сфероида, $n=0,1,2, \ldots, 0 \leqslant m \leqslant n$. Следующее утверждение доказьвает интересное свойство этих собственных значений, которое в случае шара является очевидным.

ТЕОРема 5. Собственные значения для сплюснутого (8) и вытянутого (9) сфероида удовлетворяют соотношению

$$
S_{n}(\xi):=\lambda_{n}^{0}(\xi)+2 \sum_{m=1}^{n} \lambda_{n}^{m}(\xi)=-2 \pi, \quad n=0,1, \ldots
$$

Предварительно получим некоторые полезные формулы.

Лемма 3. Справедливы следующие соотношения

$$
\begin{gathered}
\int_{-1}^{1} \frac{A_{k}(x)\left(1-x^{2}\right)^{m / 2} P_{n}^{m}(x) d x}{z-x}=2(-1)^{m} A_{k}(z)\left(z^{2}-1\right)^{m / 2} Q_{n}^{m}(z), \\
\int_{-1}^{1} \frac{\left[P_{n}^{m}(x)\right]^{2} d x}{z-x}=2(-1)^{m} P_{n}^{m}(z) Q_{n}^{m}(z), \\
P_{n}(z) Q_{n}(z)+2 \sum_{m=1}^{n}(-1)^{m} \alpha_{n}^{m} P_{n}^{m}(z) Q_{n}^{m}(z)=Q_{0}(z):=0.5 \ln \left[\frac{z+1}{z-1}\right],
\end{gathered}
$$

где $n=0,1, \ldots ; 0 \leqslant m \leqslant n, A_{k}-$ произвольный полином степени $k \leqslant n-m, z \in \mathbb{C} \backslash(-1,1)(\mathbb{C}-$ комплексная плоскость).

ДокАЗАТЕльСтво. Формулу (11) для $A_{k}(x)=x^{k}$ нам удалось найти только в справочнике [19, с. 200 , формула 8], где она приведена с опечаткой. Поэтому нам пришлось выводить ее самостоятельно, исходя из интеграла Неймана (см. [20, с. 232]):

$$
Q_{n}(z)=0.5 \int_{-1}^{1} P_{n}(t) \frac{d t}{z-t}, \quad z \in \mathbb{C} \backslash(-1,1) .
$$


Подставляя в (11) $A_{k}(x)=d^{m} P_{n}(x) / d x^{m}$ и учитьвая известные выражения для присоединенных функций Лежандра через производные от полиномов внутри и вне интервала $(-1,1)$, получаем (12). Далее, подставляя $y=x$ и $a=0$ в формулу, выражающую теорему сложения для многочленов Лежандра (см. [20, с. 233]):

$$
\begin{aligned}
& P_{n}\left\{x y+\left[\left(1-x^{2}\right)\left(1-y^{2}\right)\right]^{1 / 2} \cos a\right\} \\
& \quad=P_{n}(x) P_{n}(y)+2 \sum_{m=1}^{n} \alpha_{n}^{m} \cos (m a) P_{n}^{m}(x) P_{n}^{m}(y),
\end{aligned}
$$

с учетом $P_{n}(1)=1$ получаем

$$
P_{n}^{2}(x)+2 \sum_{m=1}^{n} \alpha_{n}^{m}\left[P_{n}^{m}(x)\right]^{2}=1 .
$$

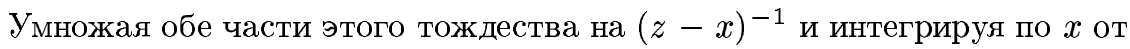
$(-1)$ до 1 , получаем, с учетом (12) и (14), основную формулу (13). Доказательство леммы завершено.

У тверждения леммы 3 позволяют легко провести

ДоКАЗАТЕЛЬСТВо ТЕОРЕМЫ 5. Подставляя значения $\lambda_{n}^{m}(\xi)$ из (8) в формулу (10), с учетом (13) получаем

$$
S_{n}(\xi)=2 \pi i\left(1+\xi^{2}\right) \frac{d[0.5 \ln ((i \xi+1) /(i \xi-1))]}{d \xi}=-2 \pi
$$

для сплюснутого сфероида. Аналогично, из формул $(10),(9)$ и (13) для вытянутого сфероида

$$
S_{n}(\xi)=2 \pi\left(\xi^{2}-1\right) \frac{d[0.5 \ln ((\xi+1) /(\xi-1))]}{d \xi}=-2 \pi,
$$

что завершает доказательство теоремы. Численные расчеты показывают, что, по-видимому, аналогичная формула справедлива не только для сфероида, но и произвольного эллипсоида.

Рассмотрим вопрос о знаках собственных чисел операторов $B$ и $B^{*}$, определенных на поверхности сфероида. Формулы (8) и (9) могут быть записаны в едином виде

$$
\lambda_{n}^{m}(z)=2 \pi\left(z^{2}-1\right) \alpha_{n}^{m}(-1)^{m} \frac{d\left\{P_{n}^{m}(z) Q_{n}^{m}(z)\right\}}{d z},
$$

где $z=\xi>1$ для вытянутого и $z=i \xi, \xi>0$, для сплюснутого сфероида. Учитьвая (12), имеем

$$
\lambda_{n}^{m}(z)=\pi \alpha_{n}^{m}\left(z^{2}-1\right) \frac{d}{d z} \int_{-1}^{1} \frac{\left[P_{n}^{m}(x)\right]^{2} d x}{z-x}, \quad z \in \mathbb{C} \backslash(-1,1) .
$$


Для вытянутого сфероида эта формула дает

$$
\lambda_{n}^{m}(\xi)=-\pi \alpha_{n}^{m}\left(\xi^{2}-1\right) \int_{-1}^{1}\left\{\frac{P_{n}^{m}(x)}{\xi-x}\right\}^{2} d x, \quad \xi>1,
$$

и мы можем повторить вывод об отрицательности всех собственных чисел операторов $B$ и $B^{*}$, сделанньй в [10]. Из (15) для сплюснутого сфероида получаем

$$
\lambda_{n}^{m}(\xi)=2 \pi\left(1+\xi^{2}\right) \alpha_{n}^{m} \int_{0}^{1} \frac{\left(x^{2}-\xi^{2}\right)\left[P_{n}^{m}(x)\right]^{2} d x}{\left(x^{2}+\xi^{2}\right)^{2}}, \quad \xi>0 .
$$

Отсюда следует, что при $\xi \geqslant 1$ все собственные значения $\lambda_{n}^{m}(\xi)$ отрицательны. Исследуя графики функций $\lambda_{n}^{m}(\xi)$ при различных $n$ и $m$, можно выдвинуть предположение, что это свойство справедливо для всех $\xi>\xi_{1}^{0} \simeq 0.660068$, однако, эта гипотеза требует строгого обоснования.

В заключение найдем явное выражение решения задачи Робена для эллипсоида. Как известно (см $[21$, с. 218$])$, эта задача сводится к отысканию собственной функции $\sigma_{0}(x)$, отвечаюшей собственному значению $\mu_{0}=-2 \pi$ оператора $B$ на поверхности $S$ или, что то же, к решению уравнения $T \sigma_{0}=$ const на $S$. Наш результат основывается на неожиданной теореме, доказанной Риттером в [22] и утверждающей следующее. Пусть $S$ представляет собой поверхность эллипсоида с полуосями $a \geqslant b \geqslant c>0$. Тогда для $p(x):=(n(x), x), x \in S$, и для любой функции $\varphi \in C(S)$ справедливо на $S$ :

$$
B(p \varphi)=p B^{*}(\varphi) .
$$

Здесь $n(x)$ - вектор единичной внешней нормали к $S$ в точке $x \in S$, а $(n(x), x)$ - соответствующее скалярное произведение в $\mathbb{R}^{3}$. Полагая в (16) $\varphi(x) \equiv 1$, легко понять, что если для некоторой поверхности $S$ вьполнено $(16)$, то функция $p(x)$ необходимо является решением задачи Робена для этой поверхности. Таким образом, справедлива

Теорема 6. Решение задачи Робена для эллипсоида имеет вид

$$
\sigma_{0}(x)=(n(x), x), \quad x \in S .
$$

Нетрудно установить явньй вид этой функции

$$
\sigma_{0}(x)=\left(\frac{x_{1}^{2}}{a^{4}}+\frac{x_{2}^{2}}{b^{4}}+\frac{x_{3}^{2}}{c^{4}}\right)^{-1 / 2}
$$

где $x=\left(x_{1}, x_{2}, x_{3}\right) \in S$. K сожалению, утверждение теоремы не обобщается на все гладкие поверхности (в частности, для тора оно не имеет места). 


\section{СПИСОК ЦИТИРОВАННОЙ ЛИТЕРАТУРЫ}

[1] Гюнтер Н. М. Теория потенциала и ее применение к основным задачам математической физики. М.: Гостехиздат, 1953.

[2] Михлин С.Г. Линейные уравнения в частных производных. М.: Высшая шткола, 1977.

[3] Смирнов В. И. Курс высшей математики. Т. 4. Ч. 2. М.: Наука, 1981.

[4] Дьедонне ЖК. Основы современного анализа. М.: Мир, 1964.

[5] Дякин В. В., Раевский В.Я. О свойствах операторов классической теории потенциала // Матем. заметки. 1989. Т. 45. № 2. С. 138-140.

[6] Агранович М.С. Дополнение к кн.: Войтович Н. Н., Каценельбаум Б.З., Сивов А.Н. Обобщенный метод собственных колебаний в теории дифракции. M.: Наука, 1977.

[7] Мазья В. Г. // Итоги науки и техн. Соврем. пробл. матем. Фундамент. направления. Т. 27. М.: ВИНИТИ, 1988. С. 131-228.

[8] Ahner J. F. Some spectral properties of an integral operator in potential theory // Proc. Edinburgh Math. Soc. 1986. V. 29. № 3. P. 405-411.

[9] Ahner J.F., Dyakin V.V., Raevskii V. Ya. New spectral results for the electrostatic integral operator // J. Math. Anal. Appl. 1994. V. 185. № 2. P. 391-402.

[10] Ahner J. F., Arenstorf R. F. On the eigenvalues of the electrostatic integral operator // J. Math. Anal. Appl. 1986. V. 117. № 1. P. 187-197.

[11] Verchota G. Layer potentials and regularity for the Dirichlet problem for Laplace's equation in Lipschitz domain // J. Funct. Anal. 1984. V. 59. № 3. P. 572-611.

[12] Белых В. Н. К проблеме численного решения задачи Дирихле гармоническим потенциалом простого слоя // Докл. РАН. 1993. Т. 329. № 4. С. 392-395.

[13] Dieudonne J. Quasi-hermitian operators // Proc. of the International Symposium on Linear Spaces. Oxford, London, New York, Jerusalem, 1961. P. $115-122$.

[14] Ерофеенко В. Т. Теоремы сложения. Минск: Наука и техника, 1989.

[15] Ahner J. F. On the eigenvalues of the electrostatic integral operator II // J. Math. Anal. Appl. 1994. V. 181. № 2. P. 328-334.

[16] Бейтмен Г., Эрдейи А. Высшие трансцендентные функции. Т. 2. М.: Наука, 1973.

[17] Абрамовиц М., Стиган И. Справочник по специальным функциям. М.: Наука, 1979.

[18] Plemelj J. Potentialtheoretische Untersuchungen // Preisschriften der Fürstlich Jablonowskischen Gesellschaft zu Leipzig. Leipzig: Teubner Verlag, 1911.

[19] Прудников А. П., Брычков Ю. А., Маричев О.И. Интегралы и ряды. Дополнительные главы. М.: Наука, 1986.

[20] Олвер $\Phi$. Введение в асимптотические методы и специальные функции. М.: Наука, 1978.

[21] Забрейко П.П., Кошелев А.И., Красносельский М.А., Михлин С.Г., Раковщик Л.С., Стеценко В.Я. Интегральные уравнения. M.: Наука, 1968.

[22] Ritter St. The spectrum of the electrostatic integral operator for an ellipsoid // Erscheint in "Methoden und Verfahren der Mathematischen Physik". Proceeding-Band der gleichnamigen Konferenz vom 12.-18.12.93 in Oberwolfach. Preprint. 